\title{
Erratum to: Validation and Comparative Studies of Four Sulfonamide Immunoassays Based on the Same Generic Polyclonal Antibody
}

\author{
Hongyan Zhang • Yan Zhang • Shuo Wang • Liguo Zang
}

Published online: 21 November 2009

(C) Humana Press 2009

\section{Erratum to: Appl Biochem Biotechnol DOI 10.1007/s12010-008-8378-x}

In the original publication, the affiliations and contact details for three of the authors were captured incorrectly. The correct information appears in the footnote.

The online version of the original article can be found under http://dx.doi.org/10.1007/s12010-008-8378-x.

H. Zhang $\cdot$ Y. Zhang $\cdot$ S. Wang $(\bowtie)$

Key Laboratory of Food Nutrition and Safety, Ministry of Education,

Faculty of Food Engineering and Biotechnology, Tianjin University of Science and Technology, Tianjin 300222, People's Republic of China

e-mail: s.wang@tust.edu.cn

L. Zang

College of Life Science, Shandong Normal University, Jinan 250014, People's Republic of China 